\title{
NumExp: Numerical epsilon expansion of hypergeometric functions
}

\author{
Zhi-Wei Huang, Jueping Liu* \\ College of Physics and Technology, Wuhan University, Wuhan 430072, China
}

\begin{abstract}
It is demonstrated that the well-regularized hypergeometric functions can be evaluated directly and numerically. The package NumExp is presented for expanding hypergeometric functions and/or other transcendental functions in a small regularization parameter. The hypergeometric function is expressed as a Laurent series in the regularization parameter and the coefficients are evaluated numerically by using the multi-precision finite difference method. This elaborate expansion method works for a wide variety of hypergeometric functions, which are needed in the context of dimensional regularization for loop integrals. The divergent and finite parts can be extracted from the final result easily and simultaneously. In addition, there is almost no restriction on the parameters of hypergeometric functions.
\end{abstract}

Keywords: Hypergeometric functions; Expansion; Feynman diagrams

\footnotetext{
* Corresponding author.

Email addresses: zwhuang@whu.edu.cn (Zhi-Wei Huang), jpliu@whu.edu.cn (Jueping Liu)

Preprint submitted to Computer Physics Communications

June 6, 2018
} 


\section{Program summary}

Program title: NumExp

Catalogue identifier:

Program summary $U R L$ :

Program obtainable from:

Licensing provisions: none

No. of lines in distributed program, including test data, etc.: 1682

No. of bytes in distributed program, including test data, etc.: 61128

Distribution format: tar.gz

Programming language: Mathematica and/or Python

Computer: Any computer where Mathematica or Python is running.

Operating system: Linux, Windows

External routines: mpmath library (for Python)

Classification: $4.4,5,11.1$

Nature of problem: Expansion of hypergeometric functions and/or other transcendental functions in a small parameter $\epsilon$. These expansions are needed in the context of dimensional regularization for loop integrals.

Solution method: The hypergeometric function is expressed as a Laurent series in the regularization parameter $\epsilon$, where the coefficients are evaluated numerically by the multi-precision finite difference method.

Restrictions: The calculation may be inefficient if the arguments of hypergeometric functions are close to the convergent boundaries.

Running time: Generally it is below a few seconds, depending on the complexity of the problem. 


\section{Introduction}

Hypergeometric functions and their extensions are used frequently in the calculation of Feynman integrals in quantum field theory [1-12]. The hypergeometric representation makes the calculation of Feynman integrals less tricky and systematic. In the past decades, several methods, such as the Mellin-Barnes techniques [1, 2, 13-15], the negative dimensional integration method (NDIM) $[16-18,7]$ or its optimized version, the method of brackets [19-22], the differential equations techniques [23], the dimensional recurrence relations $[5,10,24-26]$ and so on, have been used to obtain the hypergeometric representation of the Feynman integrals. The hypergeometric representation keeps the expression of the result in a compact form and spurious singularities cancel each other out automatically. Besides, the hypergeometric functions have good algebraic and analytic properties, which make it easy to study the physical problems in different kinematic regions.

In the context of dimensional regularization [27, 28], the space-time dimension $D=$ $4-2 \epsilon$ appears in the parameters of hypergeometric functions, where the parameter $\epsilon$ regulates infrared and/or ultraviolet divergences. Formally, the solution in the form of hypergeometric functions can be expressed as a Laurent series in $\epsilon$, but in practice this $\epsilon$-expansion of hypergeometric functions is still not a trivial task.

Recently, a number of pioneering works and elegant algorithms [29-37] devoted to construction or explicit evaluation of coefficients of analytic epsilon expansion of hypergeometric functions around integer, half-integer and rational values of parameters. Moreover, some packages like nestedsums [38, 39], Xsummer [40], HypExp [41, 42] and HYPERDIRE $[43,44]$ were developed to perform or provide helps for the analytic $\epsilon$-expansion of hypergeometric or transcendental functions. These implementations, however, are restricted to the expansion of some special classes of hypergeometric functions about integer and/or half-integer parameters. It is increasingly obvious that more general and convenient algorithms are needed to meet the demand of practical calculations.

In this work, a numerical algorithm is developed for the $\epsilon$-expansion of a wide variety of hypergeometric functions. Specifically, the generalized hypergeometric functions ${ }_{p} F_{q}$, the Appell hypergeometric functions and the Horn-type hypergeometric functions of two variables, can all be expanded in $\epsilon$ by this method. In principle hypergeometric functions are treated as mathematical objects and there is no restriction on the form of their parameters. Analytic continuation is performed automatically, which significantly simplifies the practical calculation. In addition, the regularized hypergeometric function can be evaluated numerically and lengthy expressions of the analytic expansion in terms of simpler functions are avoided. Furthermore, no knowledge of harmonic or multiple polylogarithms [45-53] or other newly defined special functions is needed. As a result, the well regularized hypergeometric functions can be treated as common functions in the practical calculations.

The numerical algorithm is based on the ansatz that the regularized hypergeometric function can be expressed as a Laurent series in the regularization parameter, where the coefficients are evaluated numerically by multi-precision finite difference method [54]. Technically, a finite small numerical parameter $\epsilon_{h}$ is introduced and the function is evaluated at a sequence of points $\epsilon_{j}=\left(j-\frac{1}{2}\right) \epsilon_{h}$, then the coefficients are approximated to order $\mathcal{O}\left(\epsilon_{h}^{n}\right)$ by optimized finite differences. In principle, there is no restriction on the order of the expansion. Obviously, parallel computation can be used to accelerate the 
numerical $\epsilon$-expansion of hypergeometric functions because function evaluations can be performed independently.

In the analytic $\epsilon$-expansion method, the coefficients of different orders are calculated separately. Usually, the expansions of $A(\epsilon)$ and $B(\epsilon)$ are needed to obtain the expansion of $A(\epsilon) B(\epsilon)$. Somewhat differently, in the numerical $\epsilon$-expansion method, all coefficients of the expansion are extracted simultaneously. In addition, $A(\epsilon) B(\epsilon)$ is expanded directly and there is no need to calculate the expansions of $A(\epsilon)$ and $B(\epsilon)$ separately. In other words, the numerical $\epsilon$-expansion method makes the expansion of complex expression fast and convenient.

The implementations of the algorithm are presented in the form of a Mathematica package NumExp.m and a Python package numexp.py. Note that the mpmath [55] package is required by the Python interface. The algorithm may also be implemented in other computation systems. Recently the NumExp package has been applied successfully to the doubly heavy hadron spectral density calculation [56], where ${ }_{q+1} F_{q}$ and/or Appell function $F_{4}$ are involved.

The calculation may be inefficient if the arguments of hypergeometric functions are close to the convergent boundaries. Convergence acceleration is used on and close to the convergent boundaries. However, the convergence acceleration may degenerate in some cases. This is an intrinsic drawback of the numerical evaluation of hypergeometric functions.

The paper is organized as follows. In the next section the theoretical background and the design of the program are described. Then the usages of the packages are shown in Sec. 3. Whereafter, some examples are presented in Sec. 4. Convergence of the algorithm near the convergent boundaries of hypergeometric functions is discussed in Sec. 5. Finally, a summary is given in Sec. 6.

\section{Theoretical background}

\subsection{Laurent expansion}

Formally, the Laurent expansion of the $\epsilon$-regularized hypergeometric function can be expressed as

$$
f(z, \epsilon)=\sum_{n=k}^{\infty} f_{n}(z) \epsilon^{n}, \quad k \in \mathbb{Z},
$$

where $f(z, \epsilon)$ is a short form of the hypergeometric function, $z$ the argument vector, and $\epsilon$ the regularization parameter, respectively. $\epsilon$ can be positive, negative or even complex. Practically, the new function $F(z, \epsilon) \equiv \epsilon^{-k} f(z, \epsilon)$ instead of $f(z, \epsilon)$ is used to perform the Laurent expansion. If $k<0$, this procedure makes the new function finite for $\epsilon \rightarrow 0$. On the other hand, if $k>0$, this procedure helps to increase the precision of the results. So, the following Laurent expansion

$$
F(z, \epsilon)=\sum_{n=-m}^{-1} \epsilon^{n} \cdot 0+\sum_{n=0}^{\infty} F_{n}(z) \epsilon^{n}
$$

will be used to derive the formulas of numerical epsilon expansion of hypergeometric functions. Note that the spuriously divergent terms $\sum_{n=-m}^{-1} \epsilon^{n} \cdot 0$ vanish for analytic 
calculation, but multi-precision computation is needed to get rid of cancellation errors from such terms for numerical calculation.

If $f\left(z, \epsilon_{1}, \epsilon_{2}\right)$ contains more than one regularization parameters, one can perform the Laurent expansions successively with respect to $\epsilon_{2}$, then $\epsilon_{1}$.

The coefficients $F_{n}(z)$ are related to the partial differentials of $F(z, \epsilon)$ with respect to $\epsilon$. In this work, the finite differences of $F(z, \epsilon)$ will be used for the numerical calculation.

\subsection{Finite difference method}

When the regularization parameter $\epsilon$ is set to zero, function $F(z, \epsilon)$ is usually illdefined. With non-positive integer parameter, Gamma function and hypergeometric function contain singularities and may not give desired results. Moreover, not only the coefficient at the leading order in $\epsilon$ is needed to be extracted. Therefore, some difference formulas like

$$
\begin{aligned}
& F_{0}(z)=F(z, \epsilon)+\mathcal{O}(\epsilon), \\
& F_{1}(z)=\frac{F(z, 2 \epsilon)-F(z, \epsilon)}{\epsilon}+\mathcal{O}(\epsilon), \\
& F_{2}(z)=\frac{F(z, 3 \epsilon)-2 F(z, 2 \epsilon)+F(z, \epsilon)}{2 ! \epsilon^{2}}+\mathcal{O}(\epsilon),
\end{aligned}
$$

and so on can be used to extract the coefficients of the Laurent expansion. That is, $F(z, 0)$ will not be used in the calculation.

The precision of the formulas above is very low. Actually, there are many ways to improve the precision and keep the computational complexity unchanged [54]. In this work, the algorithm

$$
F_{i}(z)=\sum_{j=0}^{n} \frac{C_{i j}^{n}}{n !} F\left(z,\left(j-\frac{1}{2}\right) \epsilon_{h}\right)+\mathcal{O}\left(\epsilon_{h}^{n-i+1}\right)
$$

is used for the numerical epsilon expansion. $C_{i j}^{n}$ is the $n$th degree weight array of the finite difference method, and $0 \leq i, j \leq n$. It is trivial to work out these constant arrays by solving some linear equations. For example,

$$
C_{i j}^{2}=\left(\begin{array}{rrr}
\frac{3}{8} & \frac{3}{4} & -\frac{1}{8} \\
-1 & 1 & 0 \\
1 & -2 & 1
\end{array}\right)
$$

The computation of $F\left(z,\left(j-\frac{1}{2}\right) \epsilon_{h}\right)$ is the most time-consuming part of the whole numerical calculation. It is worth noting that only $n+1$ times of function evaluation is needed to obtain all $F_{i}(z)(i=0, \ldots, n)$ to order $\mathcal{O}\left(\epsilon_{h}^{n-i}\right)$. In particular, with 3 times of function evaluation, one obtains $F_{0}(z)$ to order $\mathcal{O}\left(\epsilon_{h}^{2}\right), F_{1}(z)$ to order $\mathcal{O}\left(\epsilon_{h}^{1}\right)$ and $F_{2}(z)$ to order $\mathcal{O}\left(\epsilon_{h}^{0}\right)$, respectively. If $\epsilon_{h}$ is numerically small enough and/or $n$ is large enough, one could get the numerical $\epsilon$-expansion of $F(z, \epsilon)$ to the desired precision.

\subsection{Precision}

However, $\epsilon_{h}$ cannot be too small. From Eq. (2) and Eq. (3), it is easy to see that $-(m+n+1) \lg \left(\left|\epsilon_{h}\right|\right)$ digits of working precision is needed to obtain $F_{i}(z)$ to order $\mathcal{O}\left(\epsilon_{h}^{n-i}\right)$. 
If $\epsilon_{h}$ is too small and the working precision is insufficient, the precision of the low order coefficients is restricted by the working precision and the high order coefficients will be inaccurate or meaningless. On the other hand, higher precision means lower speed of computation. Therefore, one has to find a balance between precision and efficiency.

With a given working precision, one can use either smaller $\epsilon_{h}$ (and less function evaluations) or larger $\epsilon_{h}$ (and more function evaluations) to obtain the coefficients of the numerical $\epsilon$-expansion. For example, with 15 digits of working precision and $\epsilon_{h}=10^{-5}$, $F_{0}(z), F_{1}(z)$ and $F_{2}(z)$ have roughly 15,10 and 5 digits of precision, respectively. If $\epsilon_{h}=10^{-3}, F_{0}(z), F_{1}(z), F_{2}(z), F_{3}(z)$ and $F_{4}(z)$ have roughly $15,12,9,6$ and 3 digits of precision, respectively. It is quite obvious that lower coefficients can be evaluated to high precision even with a large $\epsilon_{h}$ at the cost of more function evaluations. It also shows that the precision of the coefficients can be increased in a trivial way.

Multi-precision computation is the key to the success of the numerical $\epsilon$-expansion method. Mathematica and the Python library mpmath [55] provide algorithms to evaluate hypergeometric functions ${ }_{p} F_{q}$ and lots of special functions to arbitrary numerical precision. In particular mpmath provides a function hyper $2 \mathrm{~d}()$ to evaluate a wide variety of hypergeometric functions of two variables, such as Appell functions $F_{1}, F_{2}, F_{3}$ and $F_{4}$, which occur in the calculations involving massive particles $[3,57,58,56]$. With the help of these computation systems, the package NumExp can be used to perform $\epsilon$-expansion of hypergeometric functions in a numerical and efficient way.

\section{Usage}

The algorithm in the preceding section is implemented in the package NumExp, and the package can be obtained from the ancillary files of arXiv:1209.3971. The package provides both Mathematica and Python interfaces for users.

\subsection{Mathematica interface}

After a successful installation, the Mathematica package NumExp.m may be loaded with the command

$$
\operatorname{In}[1]:=<<\text { NumExp } `
$$

The package provides several public functions:

- NumExpFDC [n] generates the $n$th degree weight array $C_{i j}^{n}$. If $n=2$, the array in Eq. (4) will be generated. In most cases, this function will not be used directly.

- NumExp [expr,ep,eh,n] or NumExp [expr, $\{e p, e h, n\}]$ is the main function of this package. It expands expr to order $\mathcal{O}\left(\mathrm{ep}^{n}\right)$. eh is the numerical step parameter in Eq. (3). The usage of this function is illustrated by the following example:

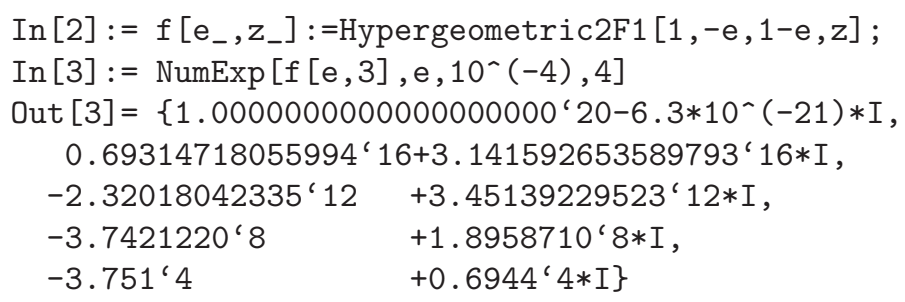


The calculation is performed with $-(n+1) \lg \left(\left|\epsilon_{h}\right|\right)$ digits of working precision and the precision of the output is estimated by Mathematica automatically. Note that the precision of the high order coefficients is slightly overestimated, which is the effect of the $\mathcal{O}\left(\epsilon_{h}^{n+1}\right)$ remainder terms. One can use smaller $\epsilon_{h}$ or take higher order expansion to increase the precision of the coefficients.

With the option: AddPrecision->np, additional $\mathrm{np}$ digits of precision is added to the working precision of NumExp. It is helpful for minimizing the effect of the spurious divergences in Eq. (2).

Besides, the precision of the input parameters must not be lower than the working precision of NumExp, or the precision of the result will be limited to the lowest precision of the input parameters.

- NumExp [expr, \{ep1, eh1,n1\}, \{ep2,eh2,n2\}] successively find numerical epsilon expansions with respect to ep2, then ep1. Explicitly,

$$
f\left(\epsilon_{1}, \epsilon_{2}\right)=\left(\begin{array}{c}
1 \\
\epsilon_{1} \\
\epsilon_{1}^{2} \\
\vdots
\end{array}\right)^{T}\left(\begin{array}{cccc}
f_{00} & f_{01} & f_{02} & \cdots \\
f_{10} & f_{11} & f_{12} & \cdots \\
f_{20} & f_{21} & f_{22} & \cdots \\
\vdots & \vdots & \vdots & \ddots
\end{array}\right)\left(\begin{array}{c}
1 \\
\epsilon_{2} \\
\epsilon_{2}^{2} \\
\vdots
\end{array}\right) .
$$

NumExp is called twice to obtain the coefficient array $\left\{f_{i j}\right\}$ and the precisions of these two expansions are assigned automatically. Usually, only the coefficients $\left\{f_{0 j}\right\}$ in the first row is of interest to users. The evaluation of the function is performed with $-\left(n_{1}+1\right) \lg \left(\left|\epsilon_{h 1}\right|\right)-\left(n_{2}+1\right) \lg \left(\left|\epsilon_{h 2}\right|\right)$ digits of working precision. In addition, the option AddPrecision can be assigned to two NumExp expansions separately and explicitly. Then additional digits of precision will be added to the working precision. This function can be used, for example, in the following way

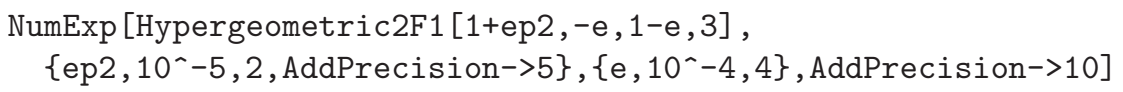

and it may be useful if auxiliary regularization parameter is used in the calculation (see Eq. (11) and Ref. [12]). It is possible and easy to extend the method to expand functions with more regularization parameters.

- ListNumExp[1st,eh,n] or ListNumExp[1st,\{eh,n\}] extracts the coefficients of the $\epsilon$-expansion from a list of values $f\left(e_{0}\right), f\left(e_{1}\right), \ldots, f\left(e_{m}\right)$ which are evaluated beforehand with $-(n+1) \lg \left(\left|\epsilon_{h}\right|\right)$ digits of working precision and the point $e_{j}=$ $\left(j-\frac{1}{2}\right) \epsilon_{h}$. eh is the numerical step parameter and $n \leq m$. The code

$$
\begin{aligned}
& \operatorname{In}[4]:=1 \text { st }=\text { Table }\left[f\left[(j-1 / 2) * 10^{\wedge}(-4), 3\right],\{j, 0,6\}\right] ; \\
& \operatorname{In}[5]:=\text { ListNumExp }\left[1 \text { st }, 10^{\wedge}(-4), 4\right]
\end{aligned}
$$

will generate the same results. If $n<m$, the first $n+1$ (from 1 to $n+1$ ) values of lst will be used by ListNumExp. Note that the step parameter eh must be consistent with the parameter in the $f\left(e_{j}\right)$ evaluations, or the expansion will be nonsense. 
This function is especially useful because the time-consuming $f\left(e_{j}\right)$ evaluations can be performed independently, which makes the parallel computation possible. Besides, one can also use other programs or packages (like mpmath [55]) to evaluate some special functions unknown to Mathematica and then use ListNumExp to obtain the epsilon expansion.

- ListNumExp [ary, \{eh1,n1\}, \{eh2,n2\}] successively find numerical epsilon expansions of the array ary with respect to ep2, then ep1. The array $\left\{f\left(e_{1 i}, e_{2 j}\right)\right\}$ is evaluated beforehand with $-\left(n_{1}+1\right) \lg \left(\left|\epsilon_{h 1}\right|\right)-\left(n_{2}+1\right) \lg \left(\left|\epsilon_{h 2}\right|\right)$ digits of precision and the point $\left(e_{1 i}, e_{2 j}\right)=\left(\left(i-\frac{1}{2}\right) \epsilon_{h 1},\left(j-\frac{1}{2}\right) \epsilon_{h 2}\right)$.

\subsection{Python interface}

Since the Python library mpmath [55] provides an extensive set of transcendental functions and these functions can be evaluated to high precision in an efficient manner, it is worth to develop a Python interface of the numerical epsilon expansion algorithm.

The Python package numexp.py is specially designed for numerical $\epsilon$-expansion of hypergeometric functions. Since $\epsilon$ is used to regularize the expression, these functions may contain spurious divergences at $\epsilon=0$ and should not be evaluated at $\epsilon=0$ directly. In the package, the function is evaluated at a list of points $\left(j-\frac{1}{2}\right) \epsilon_{h}$ and the coefficients are evaluated by finite differences of these values.

It is worth noting that mpmath [55] provides a function taylor () to produce a degree$n$ Taylor polynomial around the point $x$ of the given function $f(x)$. The differentials are approximated by finite differences and this function can handle singularity of $f(0)$ by shifting the points half of a step length $h / 2$. However, taylor () is pretty slow when the precision and the order of expansion are high because the default step parameter $h$ is related to the working precision and is too small. If a fairly large $h$ is specified, the speed increases while the precision decreases. Moreover, if the singular option is set to True, which is needed by the expansion of regularized hypergeometric functions, order $\mathcal{O}\left(n^{2}\right)$ instead of order $\mathcal{O}(n)$ algorithm is used by taylor () and the calculation is roughly $n$ times slower.

numexp. py always uses $\mathcal{O}(n)$ algorithm to calculate the coefficients of the expansion. With the given number of function evaluations, every coefficient is calculated to the best precision. The error is controlled in a systematic way and the precision is guaranteed as mentioned in Sec. 2.3. Therefore, numexp.py is recommended for numerical $\epsilon$-expansion of the regularized hypergeometric functions.

numexp.py provides two public functions:

- $\operatorname{numexp}(f, e h, n, \operatorname{args}=[])$ expands $f(e p, * a r g s)$ to order $\mathcal{O}\left(\mathrm{ep}^{n}\right)$. args is the argument list of function $f$ and eh is the numerical step parameter. The usage of this function is illustrated by the following example:

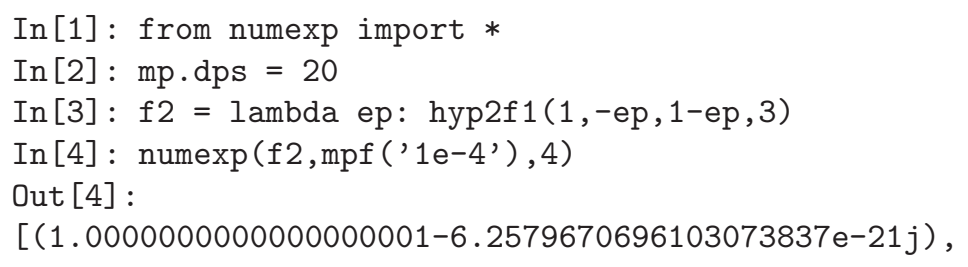




$$
\begin{aligned}
& (0.69314718055994379081+3.1415926535897933231 j), \\
& (-2.320180423351603576+3.451392295225348322 j), \\
& (-3.7421219941535409023+1.8958709608982570633 j), \\
& (-3.7510818776117221586+0.69441875410183733333 j)]
\end{aligned}
$$

These five coefficients have roughly 20, 15, 12, 7 and 3 digits of precisions. One can take the first $n-1$ coefficients and discard the last one or two. This may be an appropriate strategy for the $\epsilon$-expansion.

If the function $f()$ contains more than one argument, all other arguments except $\epsilon$ should be put in a list.

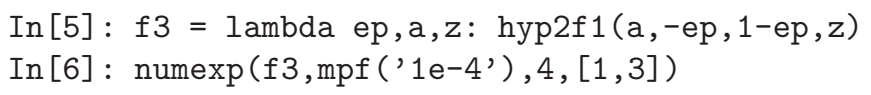

With the option: addprec=np, additional $\mathrm{np}$ digits of precision is added to the working precision of numexp. It is helpful for minimizing the effect of the spurious divergences in Eq. (2).

- listnumexp (lst, eh, n) extracts the coefficients of the $\epsilon$-expansion from a list of values $f\left(e_{0}\right), f\left(e_{1}\right), \ldots, f\left(e_{m}\right)$ which are evaluated beforehand. eh is the numerical step parameter and $n \leq m$. If $n<m$, the first $n+1$ (from 0 to $n$ ) values of 1st will be used by default.

In the following of this work, only accurate digits of the result will be kept in the results for the sake of simplicity.

\section{Examples}

In this section some examples for the numerical $\epsilon$-expansion of hypergeometric functions are given. So as to keep the expressions short, fairly large $\epsilon_{h}$ is used in the follow calculation. If the coefficients of the $\epsilon$-expansion are needed to be evaluated to a higher precision, smaller $\epsilon_{h}$ should be used.

\subsection{Hypergeometric functions and analytic continuation}

The simple Gauss hypergeometric function

$$
A(z, \epsilon)={ }_{2} F_{1}\left(\begin{array}{c}
\epsilon, \epsilon \\
1-2 \epsilon
\end{array} \mid z\right)
$$

can be analytically expanded by several packages $[39,41]$. Alternatively, it is easy to expand this function by NumExp in a numerical way. For $z=0.3$, one gets

$$
A(0.3, \epsilon)=0.999999999999999999+0.326129510 \epsilon^{2}+0.737565 \epsilon^{3}+\mathcal{O}\left(\epsilon^{4}\right),
$$

where the input parameters $\epsilon_{h}=10^{-4}$ and $n=4$ are used and only accurate digits are kept in the result. 
If the hypergeometric function is divergent for $\epsilon \rightarrow 0$, negative power terms of $\epsilon$ occur in the Laurent expansion. For the function

$$
B(z, \epsilon)={ }_{2} F_{1}\left(\begin{array}{c}
2+\epsilon, 1+\epsilon \\
-2 \epsilon
\end{array} \mid z\right),
$$

one can use $\epsilon B(z, \epsilon)$ instead of $B(z, \epsilon)$ to extract the coefficients, as stated in Sec. 2.1. Explicitly,

$$
\begin{aligned}
B(0.5, \epsilon)= & 4.00000000000000000000 \epsilon^{-1}+0.9999999999999999 \\
& +6.408403647539 \epsilon-10.5952025 \epsilon^{2}+\mathcal{O}\left(\epsilon^{3}\right) .
\end{aligned}
$$

If $|z|>1$, analytic continuation is used to evaluate the hypergeometric function. Numerically, one gets

$$
\begin{aligned}
A(3, \epsilon)= & 0.999999999999999999999+(2.320180423313-3.451392295223 i) \epsilon^{2} \\
& -(5.64797470+12.27713795 i) \epsilon^{3}-(30.5240+8.050 i) \epsilon^{4}+\mathcal{O}\left(\epsilon^{5}\right),
\end{aligned}
$$

where analytic continuation is performed automatically in the numerical calculation.

It is worth noting that analytic continuation is a non-trivial issue for the analytic expressions generated by HypExp and others [51, 42]. If one directly set $z=3$ in the analytic expansion of Eq. (5), the coefficient of $\epsilon^{4}$ term will be $-30.5241-7.35203 i$, where the imaginary part is incorrect. Technically, a small imaginary part should be introduced into the argument to keep it on the correct side of the branch cut. That is, one can use $z=3-10^{-20} i$ to reproduce the correct coefficient.

There is another issue on the analytic continuation of the hypergeometric functions. For the ${ }_{q+1} F_{q}$ functions, the analytic continuation formula for $|z|>1$ is

$$
\begin{aligned}
{ }_{q+1} F_{q}\left(\begin{array}{c}
a_{1}, \ldots, a_{q+1} \\
b_{1}, \ldots, b_{q}
\end{array} \mid z\right)= & \frac{\Gamma\left(b_{1}\right) \cdots \Gamma\left(b_{q}\right)}{\Gamma\left(a_{1}\right) \cdots \Gamma\left(a_{q+1}\right)} \sum_{i=1}^{q+1} \frac{\Gamma\left(a_{i}\right) \prod_{j=1 ; j \neq i}^{q+1} \Gamma\left(a_{j}-a_{i}\right)}{\prod_{j=1}^{q} \Gamma\left(b_{j}-a_{i}\right)} \\
& \times(-z)^{-a_{i}}{ }_{q+1} F_{q}\left(\begin{array}{c}
a_{i},\left\{1+a_{i}-b_{k}\right\}_{k=1, \ldots, q} \\
\left\{1+a_{i}-a_{k}\right\}_{k=1, \ldots, q+1 ; k \neq i}
\end{array} \mid \frac{1}{z}\right)
\end{aligned}
$$

where $a_{j}-a_{i} \notin \mathbb{Z}$. If $a_{j}-a_{i} \in \mathbb{Z}$, one can introduce another auxiliary regularization parameters $\epsilon^{\prime}$ into the parameters $a_{i}$ 's to regularize the analytically continued expression. With $a_{i} \rightarrow a_{i}+\alpha_{i} \epsilon^{\prime}$ and $\alpha_{j}-\alpha_{i} \neq 0$, the analytically continued expression is well regularized. Technically, it is advisable to use irrational $\alpha_{i}$ 's to fulfill the requirement of $\alpha_{j}-\alpha_{i} \neq 0$. Note that the expression is finite in $\epsilon^{\prime}$ and $\epsilon^{\prime}$ can be set to a small enough value in the numerical calculation.

In Ref. [56] the doubly heavy hadron spectral density has been expressed in the form 
of $\epsilon$-regularized hypergeometric functions. A part of the correlation function is

$$
\begin{aligned}
\Pi_{1}^{\text {pert }}(z)=\frac{3}{64 \pi^{6}}\{ & \frac{\Gamma(-\epsilon-4) \Gamma(-\epsilon-2)^{2} \Gamma(-\epsilon)}{\Gamma(2-\epsilon) \Gamma(-2 \epsilon-4)} \\
& \times{ }_{3} F_{2}\left(\begin{array}{c}
-\epsilon-4,-\epsilon-2+\sqrt{2} \epsilon^{\prime},-\epsilon+\sqrt{3} \epsilon^{\prime} \\
-\epsilon-\frac{3}{2}, 2-\epsilon
\end{array} \mid z\right) \\
- & \frac{\Gamma(-\epsilon-4) \Gamma(-\epsilon-2)^{2} \Gamma(-\epsilon)}{\Gamma(3-\epsilon) \Gamma(-2 \epsilon-4)} \\
& \left.\times{ }_{3} F_{2}\left(\begin{array}{c}
-\epsilon-4,-\epsilon-2+\sqrt{2} \epsilon^{\prime},-\epsilon+\sqrt{3} \epsilon^{\prime} \\
-\epsilon-\frac{3}{2}, 3-\epsilon
\end{array} \mid z\right)\right\} .
\end{aligned}
$$

The auxiliary $\epsilon^{\prime}$ is introduced to regularize the analytically continued expression. In Ref. [12], a similar regulator $\delta$ was used to cancel out the spurious divergences. By using

$$
\rho(z)=\frac{1}{2 \pi} \operatorname{Im}\{\Pi(z+i \varepsilon)-\Pi(z-i \varepsilon)\}=-\frac{1}{\pi} \operatorname{Im} \Pi(z-i \varepsilon)
$$

and setting $\epsilon^{\prime}=10^{-10}$, it is easy to extract the spectral density from the $\epsilon$-expansion of Eq. (11). One can also use the two dimensional version of NumExp (see Sec. 3) to obtain the $\epsilon^{\prime 0}$ term to a higher precision. Although the Laurent expansion of the correlation function $\Pi(z)$ contains $\epsilon^{-m}$ terms, their coefficients are all real and the imaginary parts are zero. Actually, one can directly evaluate the imaginary part of Eq. (11) to order $\mathcal{O}\left(\epsilon^{0}\right)$ and no numerical $\epsilon$-expansion is needed at all. Even so, one can use NumExp to improve the precision of the result.

Another unorthodox regularization instead of the dimensional regularization has also been used in Ref. [56] to obtain well regularized hypergeometric representation of the spectral density $\rho(z)$, where all coefficients of $\epsilon^{-m}$ terms are exactly zero. Then one can use a small $\epsilon$ to evaluate the special density directly.

\subsection{Generalized hypergeometric functions}

Integral representations of some hypergeometric functions may be used to perform the $\epsilon$-expansion. In Ref. [59], the program SecDec has been used to evaluate the $\epsilon$-expansion of two generalized hypergeometric functions

$$
\begin{aligned}
& A(\epsilon)={ }_{5} F_{4}\left(\begin{array}{c|c}
\epsilon,-\epsilon,-3 \epsilon,-5 \epsilon,-7 \epsilon & \frac{1}{2} \\
2 \epsilon, 4 \epsilon, 6 \epsilon, 8 \epsilon & 2
\end{array}\right) \\
& B(\epsilon)={ }_{4} F_{3}\left(\begin{array}{c}
-4 \epsilon,-\frac{1}{2}-\epsilon,-\frac{3}{2}-2 \epsilon, \frac{1}{2}-3 \epsilon \\
-\frac{1}{2}+2 \epsilon,-\frac{1}{2}+4 \epsilon, \frac{1}{2}+6 \epsilon
\end{array}\right) .
\end{aligned}
$$

$A(\epsilon)$ and $B(\epsilon)$ can be analytically expanded by HypExp [41, 42]. SecDec [59] takes several minutes to obtain the coefficients. It is transparent that multi-dimensional numerical integration is time-consuming and its precision is low.

The numerical epsilon expansion method might also be applied to the $\epsilon$-regularized parameter integrals, if the poles are subtracted properly. However, the precision of the multi-dimensional numerical integration is low, which makes it difficult to extract high order coefficients of the epsilon expansion by the finite difference method. 
The hypergeometric representation has the advantage in high precision calculation. NumExp takes less than one second to expand this type of generalized hypergeometric functions to order $\mathcal{O}\left(\epsilon^{6}\right)$. The numerical results are

$$
\begin{aligned}
A(\epsilon)= & 0.9999999999999999999+0.189532432184360 \epsilon \\
& -2.2990427423 \epsilon^{2}+55.469019 \epsilon^{3}-1014.39 \epsilon^{4}+\mathcal{O}\left(\epsilon^{5}\right), \\
B(\epsilon)= & 0.999999999999999999-4.27968776167885 \epsilon \\
& -26.6975474079 \epsilon^{2}+195.87119 \epsilon^{3}-7313.7 \epsilon^{4}+\mathcal{O}\left(\epsilon^{5}\right),
\end{aligned}
$$

where $\epsilon_{h}=10^{-4}$ and $n=6$ are used to perform the numerical $\epsilon$-expansion. Note that all inaccurate digits of the coefficients are discarded. The precision of the results can be improved by using smaller $\epsilon_{h}$ and/or larger $n$.

The analytic $\epsilon$-expansion of generalized hypergeometric functions about half-integer and rational parameters was studied in Refs. [33, 36, 37]. So as to illustrate the capability of NumExp, the numerical $\epsilon$-expansion of a hypergeometric function with rational and/or irrational parameters is performed here. For example, the function

$$
C(z, \epsilon)={ }_{4} F_{3}\left(\begin{array}{c}
-4 \epsilon,-\frac{1}{2}-\epsilon,-\frac{\pi}{2}-2 \epsilon, \frac{1}{3}-3 \epsilon \\
-\pi+2 \epsilon,-\frac{1}{4}+4 \epsilon, \frac{1}{2}+6 \epsilon
\end{array} \mid z\right)
$$

cannot be expanded by HypExp or other analytic $\epsilon$-expansion algorithms, while SecDec may be able to expand this function by using its integral representation. It is not surprising that NumExp can handle this kind of function with no difficulty. Explicitly,

$$
\begin{aligned}
C(0.5, \epsilon)= & 1.0000000000000000000-1.44555526747927 \epsilon \\
& +3.9383879447 \epsilon^{2}-266.94735 \epsilon^{3}+298.66 \epsilon^{4}+\mathcal{O}\left(\epsilon^{5}\right),
\end{aligned}
$$

where $\epsilon_{h}=10^{-4}$ and $n=6$ are used in the expansion.

\subsection{Hypergeometric functions of two variables}

Hypergeometric functions of two variables occur in the Feynman diagram related calculations of three kinematic variables and/or masses $[3,57,60,58,56]$. Recently, a few algorithms or packages [38-40,44] have been developed, which can be used to perform the analytic $\epsilon$-expansion of some special types of hypergeometric functions of two variables. To the authors' knowledge, these implementations can only be applied to very limited cases.

The Python library mpmath [55] can evaluate any of the 34 distinct convergent secondorder Horn-type hypergeometric series. With the help of mpmath, NumExp can be used to perform the numerical $\epsilon$-expansion of a wide variety of hypergeometric functions of two variables.

A numerical scheme was provided for the evaluation of Appell $F_{1}$ of complex parameters and real arguments in Refs. [61, 62]. Mathematica provides built-in function AppellF1 for evaluating $F_{1}$ with general parameters and arguments to arbitrary numerical precision. Practically, one can also define other Horn-type hypergeometric functions for Mathematica according to the same algorithm used by mpmath. For instance, the naive definitions of AppellF2 and AppellF4 are presented in the example file of the 
package. These functions have been used to check the correctness of the numerical epsilon expansion.

The analytic $\epsilon$-expansion of the Appell function

$$
A(x, y, \epsilon)=F_{2}\left(\begin{array}{c}
1,1, \epsilon \\
1+\epsilon, 1-\epsilon
\end{array} \mid x, y\right)
$$

is presented in Ref. [38, 39]. Here NumExp is used to show the possibility of the numerical $\epsilon$-expansion of such kind of functions. Explicitly,

$$
\begin{aligned}
A(0.3,0.4, \epsilon)= & 1.428571428571428571+0.700889880640673 \epsilon \\
& +1.6060080586 \epsilon^{2}+1.418379 \epsilon^{3}+\mathcal{O}\left(\epsilon^{4}\right), \\
A(0.3,3.4, \epsilon)= & 1.428571428571428571-(2.43800237269678+4.48798950512827 i) \epsilon \\
& -(7.0450867025-6.6246225829 i) \epsilon^{2}+(6.57967+6.095515 i) \epsilon^{3}, \quad
\end{aligned}
$$

where $\epsilon_{h}=10^{-4}$ and $n=4$ are used in the expansion. For $|x|+|y|>1$, analytic continuation is performed automatically in the numerical evaluation. The parameters of this Appell function above are pretty simple and the expression of the analytic expansion is short. If the parameters are quite complex, the expression will become lengthy and the numerical $\epsilon$-expansion may be a better choice for the practical calculation.

Presently, the analytic $\epsilon$-expansion of Appell function $F_{4}$ is valid only for some specific cases. In Ref. [12], $F_{4}$ was converted to ${ }_{2} F_{1}$ or $F_{1}$, and then XSummer [40] was used to perform the $\epsilon$-expansion. Practically, Appell $F_{4}$ and other hypergeometric functions of two variables can also be expanded by NumExp.

In the doubly heavy hadron spectral density calculation [56], Appell $F_{4}$ occurs if two heavy quarks are different. For the $(Q \bar{q})_{0}^{*}\left(\bar{Q}^{\prime} q\right)_{0}^{*}$ molecular state in Ref. [63], a part of the correlation function can be expressed as

$$
\begin{aligned}
\Pi^{\text {pert }}(s)= & \frac{9 m_{1}^{\epsilon} m_{2}^{8-\epsilon}}{256 \pi^{6}} \Gamma(2 \epsilon-4) \Gamma(-\epsilon-2) \Gamma(2-\epsilon) F_{4}\left(\begin{array}{c}
2 \epsilon-4 ;-\epsilon-2 \\
\epsilon-1,2
\end{array} \mid \frac{m_{1}^{2}}{m_{2}^{2}}, \frac{s}{m_{2}^{2}}\right) \\
& +\frac{9 m_{1}^{4-\epsilon} m_{2}^{4+\epsilon}}{256 \pi^{6}} \Gamma(\epsilon-2)^{2} \Gamma(-2 \epsilon) F_{4}\left(\begin{array}{c}
\epsilon-2 ;-2 \epsilon \\
3-\epsilon, 2
\end{array} \mid \frac{m_{1}^{2}}{m_{2}^{2}}, \frac{s}{m_{2}^{2}}\right),
\end{aligned}
$$

where the Appell $F_{4}$ functions are regularized in an unorthodox way.

For $m_{1}=1, m_{2}=2$ and $s=19$, for example, the prefactors of the first and the second terms in Eq. (22) can be expanded as

$$
\begin{aligned}
& B_{01}(\epsilon)=-10^{-5} \times\left(\frac{9.75151}{\epsilon^{2}}+\frac{9.49329}{\epsilon}+81.7264+76.8361 \epsilon\right)+\mathcal{O}\left(\epsilon^{2}\right), \\
& B_{02}(\epsilon)=-10^{-5} \times\left(\frac{7.31364}{\epsilon^{3}}+\frac{27.0103}{\epsilon^{2}}+\frac{95.1100}{\epsilon}+251.522\right)+\mathcal{O}(\epsilon),
\end{aligned}
$$

respectively. In the same way, the Appell $F_{4}$ functions of the first and the second terms in Eq. (22) can be expanded as

$$
\begin{aligned}
B_{11}(\epsilon)= & -0.75 \epsilon^{-1}+47.3086+(2.27125-6.55331 i) \epsilon \\
& -(93.1352-8.50940 i) \epsilon^{2}+\mathcal{O}\left(\epsilon^{3}\right), \\
B_{12}(\epsilon)= & 1.0+1.51042 \epsilon+(17.8362+8.73770 i) \epsilon^{2} \\
& +(0.87460-34.6270 i) \epsilon^{3}+\mathcal{O}\left(\epsilon^{4}\right),
\end{aligned}
$$


respectively. $B_{01}(\epsilon)$ need to be expanded to $\epsilon^{1}$ because $B_{11}(\epsilon)$ contains $\epsilon^{-1}$ contribution. Note that analytic continuation of $F_{4}$ is performed automatically in the numerical calculation. Then,

$$
\begin{aligned}
\Pi^{\text {pert }}(19) & =B_{01}(\epsilon) B_{11}(\epsilon)+B_{02}(\epsilon) B_{12}(\epsilon) \\
& =-\frac{0.00492267}{\epsilon^{2}}-\frac{0.00676321}{\epsilon}-(0.0380542+0.0000352703 i) .
\end{aligned}
$$

It is transparent that the imaginary parts of $\epsilon^{-m}$ terms are all zero. In fact, one can directly evaluate the imaginary part of Eq. $(22)$ to order $\mathcal{O}\left(\epsilon^{0}\right)$ and no numerical $\epsilon$ expansion is needed at all. Even so, NumExp can be used to improve the precision of the result. Moreover, the spectral density $\rho(z)$ can also be expressed by Appell $F_{4}$ functions and these $\epsilon$-regularized functions can be evaluated numerically.

\section{Convergence of the algorithm}

The calculation may be inefficient if the arguments of hypergeometric functions are close to the convergent boundaries. The standard series representation may fail to give the value of the hypergeometric function. Convergence acceleration is used on and close to the convergent boundaries. However, the convergence acceleration may degenerate in some cases. This is an intrinsic drawback of the numerical evaluation of hypergeometric functions.

For the Gauss hypergeometric function ${ }_{2} F_{1}$, the well-known analytic continuations or transformations $z \rightarrow 1-z, z \rightarrow z /(z-1), z \rightarrow 1 / z, z \rightarrow 1-1 / z$ and $z \rightarrow 1 /(1-z)$ all $\operatorname{map} z=e^{ \pm i \pi / 3}$ into $z=e^{ \pm i \pi / 3}$. That is, these two points are always on the convergent boundary, where the series converges slowly, or not at all. There are recurrence algorithms for evaluating ${ }_{2} F_{1}$ in the vicinity of these special points [64,65]. Mathematica and mpmath [55] have similar strategies to evaluate ${ }_{2} F_{1}$ near these points and the speed is fairly fast.

As for the generalized hypergeometric functions ${ }_{q+1} F_{q}$, the well-known analytic continuation is $z \rightarrow 1 / z$ and $|z|=1$ is the convergent boundary. Convergence acceleration is used on and close to the unit circle. However, the convergence acceleration may degenerate as $|z-1| \rightarrow 0$. Mathematica can give the result to high precision, though the speed might be a little slow. Recently, some algorithms for evaluating ${ }_{q+1} F_{q}$ near $z=1$ has been presented [66-71]. These algorithms may be helpful for improving and accelerating the evaluation of ${ }_{q+1} F_{q}$ near $z=1$.

Numerical evaluation of two-variable hypergeometric functions is even complicated and time-consuming. Presently, only Appell $F_{1}$ is well-implemented to some extent. The other Horn-type hypergeometric functions can be evaluated by rewriting the series so that the inner series has the form of an ordinary generalized hypergeometric function and thereby can be evaluated efficiently [55]. The inner generalized hypergeometric function can be analytically continued to other regions. Consequently, the two-variable hypergeometric function is analytically continued. Obviously, the convergent boundaries are complicated. If the variables are close the convergent boundaries, the series representation may take quite a long time or even fail to give the correct result. Inevitably NumExp will be inefficient for such cases because NumExp highly relies on the multiple-precision evaluation of hypergeometric functions. In view of the fact that the analytic epsilon expansion of two-variable hypergeometric functions is available only for some special cases, NumExp can still be very useful in the practical calculations. 
Actually, the users can employ fast algorithms or programs to evaluate the hypergeometric function, and then use ListNumExp or listnumexp of NumExp to extract the coefficients of epsilon expansion. The most time-consuming function evaluations can be performed in a totally parallel way, which makes the numerical epsilon expansion method of practical uses even when the hypergeometric function evaluation is pretty slow.

\section{Summary}

The Feynman integrals can be calculated in a less tricky and systematic way by using the hypergeometric representation. Presently, the analytic $\epsilon$-expansion methods of hypergeometric functions have been used in practice. However, the application of such expansion has lots of limitations. For example, only some classes of hypergeometric functions with the specific form of parameters can be expanded, and some newly defined special functions are used as primary elements and the expanded expressions are quite lengthy which make the physical analysis cumbersome.

In this work it is demonstrated that the well-regularized hypergeometric functions can be evaluated directly and numerically. An algorithm is developed and the package NumExp is presented for expanding hypergeometric functions and/or other transcendental functions in a small regularization parameter. Hypergeometric function is expressed as a Laurent series in the regularization parameter and the coefficients are evaluated numerically by using multi-precision finite difference method. This elaborate expansion method works for a wide variety of (hypergeometric) functions, such as the generalized hypergeometric functions ${ }_{p} F_{q}$ (not just the ${ }_{q+1} F_{q}$ ), the Appell hypergeometric functions and other Horn-type hypergeometric functions of two variables, which are needed in the context of dimensional regularization for loop integrals. It is straightforward and easy to obtain the high order coefficients $F_{n}(z)$ of the numerical $\epsilon$-expansion. In addition, there is almost no restriction on the form of parameters of hypergeometric functions.

The numerical $\epsilon$-expansion method may not be suitable for the intermediate expressions, but it is good enough for the final results. Practically, the divergent and finite parts can be extracted from the final result easily and simultaneously. Moreover, parallel computation can be used to accelerate the numerical $\epsilon$-expansion of hypergeometric functions. In other words, the numerical $\epsilon$-expansion method makes the evaluation of Feynman integrals fast and convenient.

It is possible and easy to extend the method to expand functions with two or more regularization parameters. It is worth noting that this method can serve to be an important cross-check of the analytic $\epsilon$-expansion methods and it can be used in case of the analytic $\epsilon$-expansion is not available.

\section{Acknowledgments}

We thank the anonymous referees for providing constructive comments and help in improving the contents of this paper. We are also grateful to Mikhail Kalmykov for his technical comments on our work. We would like to thank Ze-kun Guo for careful reading of the manuscript. This work is supported by the National Natural Science Foundation of China under Grant No. 10775105, BEPC National Laboratory Project R\&D and BES Collaboration Research Foundation, and the project of Wuhan University of China under the Grant No. 201103013 and 9yw201115. 


\section{References}

[1] E. Boos, A. I. Davydychev, A Method of evaluating massive Feynman integrals, Theor.Math.Phys. 89 (1991) 1052-1063. doi:10.1007/BF01016805, 10.1007/BF01016805.

[2] A. I. Davydychev, General results for massive N point Feynman diagrams with different masses, J.Math.Phys. 33 (1992) 358-369. doi:10.1063/1.529914.

[3] A. I. Davydychev, J. Tausk, Two loop selfenergy diagrams with different masses and the momentum expansion, Nucl.Phys. B397 (1993) 123-142. doi:10.1016/0550-3213(93) 90338-P.

URL http://www.higgs.de/ davyd/preprints/dt1.pdf

[4] A. I. Davydychev, M. Y. Kalmykov, New results for the epsilon expansion of certain one, two and three loop Feynman diagrams, Nucl.Phys. B605 (2001) 266-318. arXiv:hep-th/0012189, doi:10.1016/S0550-3213(01)00095-5.

[5] O. Tarasov, Connection between Feynman integrals having different values of the space-time dimension, Phys.Rev. D54 (1996) 6479-6490. arXiv:hep-th/9606018, doi:10.1103/PhysRevD.54.6479.

[6] C. Anastasiou, E. W. N. Glover, C. Oleari, Application of the negative-dimension approach to massless scalar box integrals, Nucl. Phys. B565 (2000) 445-467. arXiv:hep-ph/9907523, doi:10.1016/S0550-3213(99)00636-7.

[7] C. Anastasiou, E. W. N. Glover, C. Oleari, Scalar One-Loop Integrals using the Negative-Dimension Approach, Nucl. Phys. B572 (2000) 307-360. arXiv:hep-ph/9907494 doi:10.1016/S0550-3213(99)00637-9.

[8] C. Anastasiou, E. W. N. Glover, C. Oleari, The two-loop scalar and tensor pentabox graph with light- like legs, Nucl. Phys. B575 (2000) 416-436. arXiv:hep-ph/9912251, doi:10.1016/S0550-3213(00)00079-1.

[9] T. Gehrmann, E. Remiddi, Differential equations for two loop four point functions, Nucl.Phys. B580 (2000) 485-518. arXiv:hep-ph/9912329, doi:10.1016/S0550-3213(00)00223-6.

[10] J. Fleischer, F. Jegerlehner, O. Tarasov, A New hypergeometric representation of one loop scalar integrals in d dimensions, Nucl.Phys. B672 (2003) 303-328. arXiv:hep-ph/0307113, doi: $10.1016 / j$.nuclphysb.2003.09.004.

[11] S. Weinzierl, Subtraction terms at NNLO, JHEP 0303 (2003) 062 arXiv:hep-ph/0302180, doi: $10.1088 / 1126-6708 / 2003 / 03 / 062$.

[12] V. Del Duca, C. Duhr, E. Nigel Glover, V. A. Smirnov, The One-loop pentagon to higher orders in epsilon, JHEP 1001 (2010) 042. arXiv:0905.0097, doi:10.1007/JHEP01(2010) 042.

[13] V. A. Smirnov, Analytical result for dimensionally regularized massless on shell double box, Phys.Lett. B460 (1999) 397-404. arXiv:hep-ph/9905323, doi:10.1016/S0370-2693(99) 00777-7.

[14] J. Tausk, Nonplanar massless two loop Feynman diagrams with four on-shell legs, Phys.Lett. B469 (1999) 225-234. arXiv:hep-ph/9909506, doi:10.1016/S0370-2693(99)01277-0.

[15] M. Y. Kalmykov, B. A. Kniehl, Mellin-Barnes representations of Feynman diagrams, linear systems of differential equations, and polynomial solutions, Phys.Lett. B714 (2012) 103-109. arXiv:1205.1697, doi:10.1016/j.physletb.2012.06.045.

[16] I. Halliday, R. Ricotta, NEGATIVE DIMENSIONAL INTEGRALS. 1. FEYNMAN GRAPHS, Phys.Lett. B193 (1987) 241. doi:10.1016/0370-2693(87)91229-9.

[17] A. T. Suzuki, A. G. M. Schmidt, Two-loop self-energy diagrams worked out with NDIM, Eur. Phys. J. C5 (1998) 175-179. arXiv:hep-th/9709144, doi:10.1007/s100520050259.

[18] A. T. Suzuki, A. G. M. Schmidt, Negative-dimensional integration revisited, J. Phys. A31 (1998) 8023-8039. doi:10.1088/0305-4470/31/39/015.

[19] I. Gonzalez, I. Schmidt, Optimized Negative Dimensional Integration Method (NDIM) and multiloop Feynman diagram calculation, Nucl. Phys. B769 (2007) 124-173. arXiv:hep-th/0702218, doi:10.1016/j.nuclphysb.2007.01.031.

[20] I. Gonzalez, V. H. Moll, Definite integrals by the method of brackets. Part 1arXiv:0812.3356.

[21] I. Gonzalez, V. Moll, I. Schmidt, A generalized Ramanujan Master Theorem applied to the evaluation of Feynman diagramsarXiv: 1103.0588

[22] I. Gonzalez, V. H. Moll, A. Straub, The Method of brackets. Part 2. Examples and applicationsarXiv: 1004.2062.

[23] A. Kotikov, Differential equations method: New technique for massive Feynman diagrams calculation, Phys.Lett. B254 (1991) 158-164. doi:10.1016/0370-2693(91) 90413-K.

[24] O. Tarasov, Hypergeometric representation of the two-loop equal mass sunrise diagram, Phys.Lett. B638 (2006) 195-201. arXiv:hep-ph/0603227, doi:10.1016/j.physletb.2006.05.033.

[25] R. N. Lee, Space-time dimensionality D as complex variable: calculating loop integrals using di- 
mensional recurrence relation and analytical properties with respect to D, Nucl. Phys. B830 (2010) 474-492. arXiv:0911.0252, doi:10.1016/j.nuclphysb.2009.12.025.

[26] B. A. Kniehl, O. V. Tarasov, Analytic result for the one-loop scalar pentagon integral with massless propagators, Nucl.Phys. B833 (2010) 298-319. arXiv:1001.3848, doi:10.1016/j.nuclphysb.2010.03.006.

[27] G. 't Hooft, M. Veltman, Regularization and Renormalization of Gauge Fields, Nucl.Phys. B44 (1972) 189-213. doi:10.1016/0550-3213(72) 90279-9.

[28] C. Bollini, J. Giambiagi, Dimensional Renormalization: The Number of Dimensions as a Regularizing Parameter, Nuovo Cim. B12 (1972) 20-25.

[29] M. Y. Kalmykov, O. Veretin, Single scale diagrams and multiple binomial sums, Phys.Lett. B483 (2000) 315-323. arXiv:hep-th/0004010, doi:10.1016/S0370-2693(00)00574-8.

[30] F. Jegerlehner, M. Y. Kalmykov, O. Veretin, MS-bar versus pole masses of gauge bosons. 2. Two loop electroweak fermion corrections, Nucl.Phys. B658 (2003) 49-112. arXiv:hep-ph/0212319, doi:10.1016/S0550-3213(03)00177-9.

[31] F. Jegerlehner, M. Y. Kalmykov, $\mathrm{O}($ alpha alpha(s)) correction to the pole mass of the $t$ quark within the standard model, Nucl.Phys. B676 (2004) 365-389. arXiv:hep-ph/0308216, doi:10.1016/j.nuclphysb.2003.10.012.

[32] A. I. Davydychev, M. Y. Kalmykov, Massive Feynman diagrams and inverse binomial sums, Nucl. Phys. B699 (2004) 3-64. arXiv:hep-th/0303162, doi:10.1016/j.nuclphysb.2004.08.020.

[33] S. Weinzierl, Expansion around half integer values, binomial sums and inverse binomial sums, J.Math.Phys. 45 (2004) 2656-2673. arXiv:hep-ph/0402131, doi:10.1063/1.1758319.

[34] M. Y. Kalmykov, B. F. L. Ward, S. Yost, All order epsilon-expansion of Gauss hypergeometric functions with integer and half/integer values of parameters, JHEP 02 (2007) 040 arXiv:hep-th/0612240, doi:10.1088/1126-6708/2007/02/040

[35] M. Y. Kalmykov, Gauss hypergeometric function: Reduction, epsilon- expansion for integer / half-integer parameters and Feynman diagrams, JHEP 04 (2006) 056. arXiv:hep-th/0602028, doi: 10.1088/1126-6708/2006/04/056.

[36] M. Y. Kalmykov, B. F. L. Ward, S. A. Yost, Multiple (inverse) binomial sums of arbitrary weight and depth and the all-order epsilon-expansion of generalized hypergeometric functions with one half-integer value of parameter, JHEP 10 (2007) 048. arXiv:0707.3654, doi:10.1088/1126-6708/2007/10/048.

[37] M. Y. Kalmykov, B. A. Kniehl, Towards all-order Laurent expansion of generalized hypergeometric functions around rational values of parameters, Nucl. Phys. B809 (2009) 365-405. arXiv:0807.0567, doi:10.1016/j.nuclphysb.2008.08.022.

[38] S. Moch, P. Uwer, S. Weinzierl, Nested sums, expansion of transcendental functions and multi-scale multi-loop integrals, J. Math. Phys. 43 (2002) 3363-3386. arXiv:hep-ph/0110083, doi:10.1063/1.1471366.

[39] S. Weinzierl, Symbolic Expansion of Transcendental Functions, Comput. Phys. Commun. 145 (2002) 357-370. arXiv:math-ph/0201011, doi:10.1016/S0010-4655(02)00261-8.

[40] S. Moch, P. Uwer, XSummer: Transcendental functions and symbolic summation in Form, Comput. Phys. Commun. 174 (2006) 759-770. arXiv:math-ph/0508008, doi:10.1016/j.cpc.2005.12.014.

[41] T. Huber, D. Maitre, HypExp, a Mathematica package for expanding hypergeometric functions around integer-valued parameters, Comput. Phys. Commun. 175 (2006) 122-144. arXiv:hep-ph/0507094, doi:10.1016/j.cpc.2006.01.007.

[42] T. Huber, D. Maitre, HypExp 2, Expanding Hypergeometric Functions about Half- Integer Parameters, Comput. Phys. Commun. 178 (2008) 755-776. arXiv:0708.2443, doi:10.1016/j.cpc.2007.12.008.

[43] M. Y. Kalmykov, B. F. L. Ward, S. A. Yost, On the all-order epsilon-expansion of generalized hypergeometric functions with integer values of parameters, JHEP 11 (2007) 009. arXiv:0708.0803, doi:10.1088/1126-6708/2007/11/009.

[44] V. V. Bytev, M. Y. Kalmykov, B. A. Kniehl, HYPERDIRE: HYPERgeometric functions DIfferential REduction MATHEMATICA based packages for differential reduction of generalized hypergeometric functions: Now with ${ }_{p} F_{p-1}, F_{1}, F_{2}, F_{3}, F_{4}$ arXiv: 1105.3565.

[45] J. Vermaseren, Harmonic sums, Mellin transforms and integrals, Int.J.Mod.Phys. A14 (1999) $2037-$ 2076. arXiv:hep-ph/9806280, doi:10.1142/S0217751X99001032

[46] E. Remiddi, J. A. M. Vermaseren, Harmonic polylogarithms, Int. J. Mod. Phys. A15 (2000) $725-754$ arXiv: hep-ph/9905237, doi:10.1142/S0217751X00000367.

[47] T. Gehrmann, E. Remiddi, Numerical evaluation of harmonic polylogarithms, Comput.Phys.Commun. $141 \quad$ (2001) 296-312. arXiv:hep-ph/0107173, 
doi:10.1016/S0010-4655(01)00411-8.

[48] T. Gehrmann, E. Remiddi, Numerical evaluation of two-dimensional harmonic polylogarithms, Comput.Phys.Commun. 144 (2002) 200-223. arXiv:hep-ph/0111255, doi : 10.1016/S0010-4655(02)00139-X.

[49] J. Vollinga, S. Weinzierl, Numerical evaluation of multiple polylogarithms, Comput.Phys.Commun. 167 (2005) 177. arXiv:hep-ph/0410259, doi:10.1016/j.cpc.2004.12.009.

[50] D. Maitre, HPL, a Mathematica implementation of the harmonic polylogarithms, Comput. Phys. Commun. 174 (2006) 222-240. arXiv:hep-ph/0507152, doi:10.1016/j.cpc.2005.10.008.

[51] D. Maitre, Extension of HPL to complex arguments, Comput.Phys.Commun. 183 (2012) 846 arXiv:hep-ph/0703052, doi:10.1016/j.cpc.2011.11.015.

[52] R. Bonciani, G. Degrassi, A. Vicini, On the Generalized Harmonic Polylogarithms of One Complex Variable, Comput.Phys.Commun. 182 (2011) 1253-1264. arXiv:1007.1891, doi:10.1016/j.cpc.2011.02.011.

[53] S. Buehler, C. Duhr, CHAPLIN - Complex Harmonic Polylogarithms in FortranarXiv: 1106.5739.

[54] B. Fornberg, Generation of Finite Difference Formulas on Arbitrarily Spaced Grids, Mathematics of Computation 51 (1988) 699-706. doi:10.2307/2008770.

[55] F. Johansson, et al., mpmath: a Python library for arbitrary-precision floating-point arithmetic (version 0.17) (2012).

URL http://code.google.com/p/mpmath/

[56] Z.-W. Huang, J. Liu, Analytic calculation of doubly heavy hadron spectral density in coordinate spacearXiv: 1205.3026 .

[57] M. Shpot, A Massive Feynman integral and some reduction relations for Appell functions, J.Math.Phys. 48 (2007) 123512. arXiv:0711.2742, doi:10.1063/1.2821256.

[58] B. A. Kniehl, O. V. Tarasov, Finding new relationships between hypergeometric functions by evaluating Feynman integrals, Nucl.Phys. B854 (2012) 841-852. arXiv:1108.6019, doi:10.1016/j.nuclphysb.2011.09.015.

[59] J. Carter, G. Heinrich, SecDec: A general program for sector decomposition, Comput.Phys.Commun. 182 (2011) 1566-1581. arXiv:1011.5493, doi:10.1016/j.cpc.2011.03.026.

[60] R. Alkofer, M. Q. Huber, K. Schwenzer, Infrared Behavior of Three-Point Functions in Landau Gauge Yang-Mills Theory, Eur.Phys.J. C62 (2009) 761-781. arXiv:0812.4045, doi: 10.1140/epjc/s10052-009-1066-3.

[61] F. Colavecchia, G. Gasaneo, J. Miraglia, Numerical evaluation of Appell's $F_{1}$ hypergeometric function, Comput.Phys.Commun. 138 (2001) 29-43. doi:10.1016/S0010-4655(01)00186-2.

[62] F. Colavecchia, G. Gasaneo, f1: a code to compute Appell's $F_{1}$ hypergeometric function, Comput.Phys.Commun. 157 (2004) 32-38. doi:10.1016/S0010-4655(03)00490-9.

[63] J.-R. Zhang, M.-Q. Huang, $\{Q \bar{q}\}\left\{\bar{Q}^{\left({ }^{\prime}\right)} q\right\}$ molecular states, Phys.Rev. D80 (2009) 056004. arXiv:0906.0090, doi:10.1103/PhysRevD.80.056004.

[64] W. Bühring, An analytic continuation of the hypergeometric series, SIMA J. Math. Anal. 18 (1988) 884889. doi:10.1137/0518066

[65] N. Michel, M. Stoitsov, Fast computation of the Gauss hypergeometric function with all its parameters complex with application to the Poschl-Teller-Ginocchio potential wave functions, Comput. Phys. Commun. 178 (2007) 535551. arXiv:0708.0116, doi:10.1016/j.cpc.2007.11.007.

[66] W. Bühring, Generalized hypergeometric functions at unit argument, Proc. Amer. Math. Soc. 114 (1992) 145-153. doi:10.2307/2159793.

[67] W. Bühring, H. M. Srivastava, Analytic continuation of the generalized hypergeometric series near unit argument with emphasis on the zero-balanced seriesarXiv:math/0102032 .

[68] W. Bühring, Partial sums of hypergeometric series of unit argument, Proc. Amer. Math. Soc. 132 (2004) 407-415. arXiv:math/0311126

[69] S. Skorokhodov, A method for computing the generalized hypergeometric function ${ }_{p} F_{p-1}\left(a_{1}, \ldots, a_{p} ; b_{1}, \ldots, b_{p-1} ; 1\right)$ in terms of the riemann zeta function, Comput. Math. Math. Phys. 45 (2005) 550-562.

[70] A. I. Bogolubsky, S. L. Skorokhodov, Fast evaluation of the hypergeometric function ${ }_{p} F_{p-1}(a ; b ; z)$ at the singular point $z=1$ by means of the Hurwitz zeta function $\zeta(\alpha, s)$, Programming and Computing Software 32 (2006) 145-153. doi:10.1134/S0361768806030054.

[71] J. L. Willis, Acceleration of generalized hypergeometric functions through precise remainder asymptotics, Num. Alg. 59 (2012) 447-485. arXiv:1102.3003, doi:10.1007/s11075-011-9499-9. 